Chapter 2

\title{
Wireless Coded Deadbeat Power Control for Wind Energy Generation
}

\author{
C. E. Capovilla, A. J. Sguarezi Filho, I. R. S. Casella and \\ E. Ruppert
}

Additional information is available at the end of the chapter

http://dx.doi.org/10.5772/54687

\section{Introduction}

The growing demand for energy by the developing and developed countries, the search for energy alternatives to the use of fossil fuel and the recent special attention given to the environment, makes the study of alternative and renewable generation sources of electric energy in power grids extremely important [1].

Recently, the renewable power grids that carry electricity generated by wind, solar, and tidal have received new investments to turn out to be feasible and to optimize their use based on the concept of smart grids [2]. Among all sources of electric energy applied to this new concept, wind generation has emerged as one of the most promising presented techniques and has been the focus of several recent scientific studies [3, 4].

For a successful implementation, it is necessary to develop a complete telecommunications framework composed by communication networks, data management, and real-time monitoring applications with a strong interaction. In particular, the application of a modern telecommunication system for controlling and monitoring in smart grids applications requires a complex infrastructure for an efficient operation [5], and its development and operability presents several non-trivial issues due to the convergence of different areas of knowledge and design aspects.

In this way, wireless communications appear as an interesting solution for offering many benefits such as low cost of development, expansion facilities, possibility of using the technologies currently applied in mobile telephone systems, flexibility of use, and distributed management. However, wireless transmissions are subject to distortions and errors caused by the propagation channel that can cause serious problems to the controlled and monitored equipments, thus, to the energy plant as a whole. This intrinsic problem of wireless communication systems can be circumvented through the use of Forward Error Correction 
(FEC) [6]. This coding technique is used in all modern wireless digital systems and is essential to ensure the integrity of information, reducing significantly the Bit Error Rate (BER) and the latency of the information by adding redundancy to the transmitted information [7].

There are currently several different schemes of FEC that are used in commercial wireless communication systems, for instance, the Reed Solomon (RS) coding [8], Convolutional Coding (CONV) [9], Turbo Coding (TC) [10, 11], and Low Density Parity Check (LDPC) coding [12-14]. Among them, the LDPC coding is the one that presents the best performance, approaching significantly to the limits set by the seminal work of Shannon [15] and that shows an excellent compromise between decoding complexity and performance [13, 16]. Besides, the LDPC coding has recently been added to the IEEE 802.16e Standard, commonly known as Worldwide Interoperability for Microwave Access (WiMAX) for mobile applications [17].

It is worth noting that there are some works in the scientific literature referencing the application of wireless technology for monitoring wind energy systems based on sensor networks [18, 19], however there has not been presented yet any deep research about the use of wireless technology for control applications in these systems, making it difficult to estimate the real impact of its use or its advantages and difficulties.

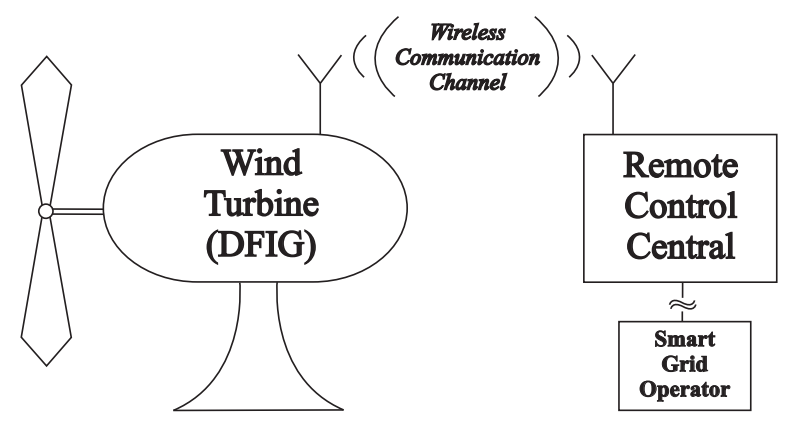

Figure 1. Wireless System Control Schematic.

Concerning the generators used in wind turbines, the Doubly-Fed Induction Generators (DFIG) have been widely used in these systems [20] due to their great characteristics. The main advantages of using DFIG are their ability to operate at variable speeds and control the active and reactive power into four quadrants, in contrast, for instance, to the Squirrel Cage Induction Generators (SCIG), which operate at fixed speed [1, 21]. The active and reactive power control of DFIG is made by using field orientation. In the work [22], some investigations were carried out to power control of a DFIG using Proportional-Integral (PI) controller, however this type of controller has problems related to the design of their gain due to operating conditions of the generator. In the works [23], [24], and [25] other investigations were done, respectively, for the use of predictive control techniques and internal mode control. Although both controllers show a satisfactory performance, they present many difficulties to implementation due to their intrinsic formulations.

In this context, this work proposes a wireless coding deadbeat power control using DFIG machine to improve robustness and reliability of the generation system, as shown in Fig. 1 
(simplified schematic). The proposed controller is based on discrete dynamic mathematical model of the generator and it uses the vector control technique that allows the independent control of active and reactive power.

The wireless communication system is used to send the power reference signals to the DFIG controller applying an LDPC coding scheme to reduce the transmission errors and the overall system latency. The performance of the proposed system is investigated for different radio propagation scenarios to evaluate the real impact of the wireless transmission in the wind energy control system. It is noteworthy that the errors generated in the wireless transmission cannot be easily removed without using advanced FEC coding techniques similar to those presented in this work.

Although the proposed system has been analyzed for a single link between one remote control unit and one aerogenerator, it can easily be expanded to control several aerogenerators for wind farm applications. The use of wireless communication in wind farms becomes very interesting for technical and economic reasons. The work of [26] shows a wireless remote control for a wind farm consisting of offshore wind generation platforms. The choice of an appropriate control system and a wireless monitoring becomes essential for this type of application, due to its easy deployment avoiding the need for submarine optical fibers that have high cost of installation and maintenance. Besides, any changes in the offshore platforms positioning due to climate or hydrological characteristics would not be problem for the wireless communication system. The importance of that the communication systems have on the effective control and maintenance of wind farms is discussed in the work of [27]. A brief description of IEC61400-25 "Communications for Monitoring and Control of Wind Power Plants" is provided. As a case study, it was analyzed the Horns Rev offshore wind farm in Denmark that employs a principal communication system based on optical fiber and a secondary wireless system, both integrated into the Supervisory Control And Data Acquisition (SCADA) system, linking wind turbines to the onshore control center. Additionally, the work of [28] has shown some problems present in a real wired system to control and monitor wind turbines based on Lonworks and the authors present as solution a wireless control and monitoring system that offers many facility and benefits.

These works bring evidences and exemplify the actual advantages and features offered by the use of wireless communications, but none of them proposes or examines techniques that can ensure the reliability and security for control and monitoring information on transmission error robustness, due to the degrading effects of wireless communication channel. Thus, this work aims to fill a gap in the literature to demonstrate the functional viability of the use of wireless systems for this type of application when an appropriate coding technique is applied.

The chapter is organized as follows: DFIG adaptive deadbeat power control is shown in section 2, the wireless coding communication is presented in section 3, main results are considered in section 4 , and section 5 concludes the chapter.

\section{DFIG Deadbeat power control}

The doubly-fed induction machine in synchronous reference frame can be represented [29] by: 


$$
\begin{gathered}
\vec{v}_{1 d q}=R_{1} \vec{i}_{1 d q}+\frac{d \vec{\lambda}_{1 d q}}{d t}+j \omega_{1} \vec{\lambda}_{1 d q} \\
\vec{v}_{2 d q}=R_{2} \vec{i}_{2 d q}+\frac{d \vec{\lambda}_{2 d q}}{d t}+j\left(\omega_{1}-B \omega_{r}\right) \vec{\lambda}_{2 d q}
\end{gathered}
$$

where: $\vec{v}_{d q}, \vec{i}_{d q}, \vec{\lambda}_{d q}$ are, respectively, voltage, current, and flux space vectors in synchronous reference frame $d q, R$ is the resistance of the winding, $L$ is the inductance of the winding, $B$ is the number of pairs of poles, $\omega_{1}$ is the synchronous speed, $\omega_{r}$ is the rotor speed, and the subscripts 1 and 2 denote, respectively, stator and rotor parameters.

The generator active and reactive power can be obtained by:

$$
\begin{aligned}
& P=\frac{3}{2}\left(v_{1 d} i_{1 d}+v_{1 q} i_{1 q}\right) \\
& Q=\frac{3}{2}\left(v_{1 q} i_{1 d}-v_{1 d} i_{1 q}\right)
\end{aligned}
$$

Using stator flux oriented control, that decouples $d$ and $q$ components, the relationship between stator and rotor currents becomes:

$$
\begin{gathered}
i_{1 d}=\frac{\lambda_{1}}{L_{1}}-\frac{L_{M}}{L_{1}} i_{2 d} \\
i_{1 q}=-\frac{L_{M}}{L_{1}} i_{2 q}
\end{gathered}
$$

where: $v, i, \lambda$ are, respectively, voltage, current and flux magnitudes and $L_{M}$ is the mutual inductance.

The active (3) and reactive (4) power can be calculated by using (5) and (6) and it is given by:

$$
\begin{gathered}
P=-\frac{3}{2} v_{1} \frac{L_{M}}{L_{1}} i_{2 q} \\
Q=\frac{3}{2} v_{1}\left(\frac{\lambda_{1}}{L_{1}}-\frac{L_{M}}{L_{1}} i_{2 d}\right)
\end{gathered}
$$

Equations (8) and (7) show the system can provide independent active and reactive power control by regulating the rotor current. The proposed deadbeat power control, shown in 
Fig. 2, considers these relationships. Consequently, stator active and reactive power control can be accomplished by using rotor current control of the DFIG with stator directly connected to the grid.

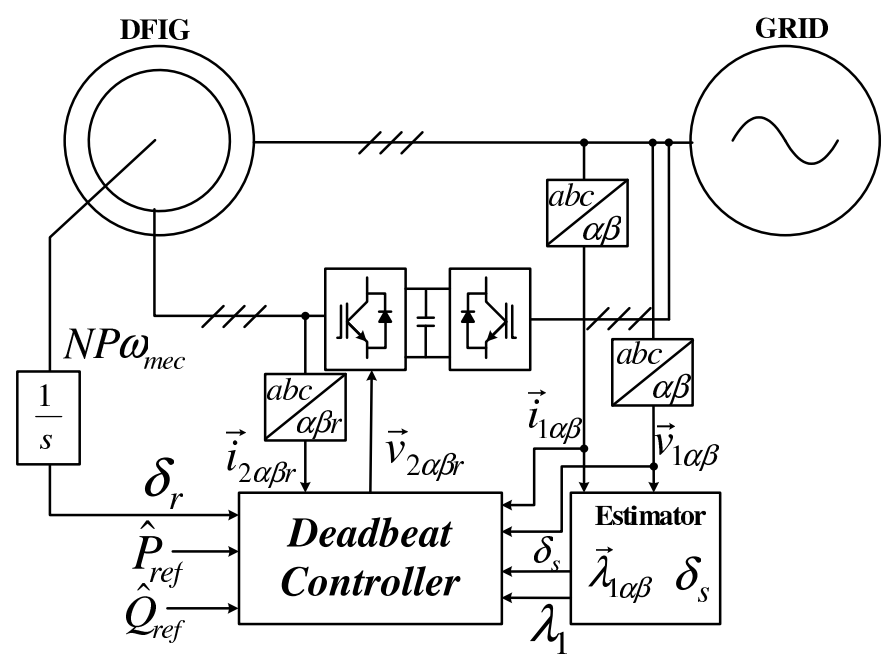

Figure 2. Deadbeat Power Control Block Diagram.

The discretized rotor equation (based on the zero-order hold method) in the synchronous referential frame, using equation (2), the stator flux position at sampling time $k+1$ and using equations (5) and (6), can be represented [30] by:

$$
\begin{array}{r}
{\left[\begin{array}{l}
i_{2 d}(k+1) \\
i_{2 q}(k+1)
\end{array}\right]=\left[\begin{array}{cc}
1-\frac{R_{2} T}{\sigma L_{2}} & \frac{\omega_{s l} T}{\sigma} \\
\frac{-\omega_{s l} T}{\sigma} & 1-\frac{R_{2} T}{\sigma L_{2}}
\end{array}\right]\left[\begin{array}{l}
i_{2 d}(k) \\
i_{2 q}(k)
\end{array}\right]+} \\
+\left[\begin{array}{cc}
\frac{T}{\sigma L_{2}} & 0 \\
0 & \frac{T}{\sigma L_{2}}
\end{array}\right]\left[\begin{array}{l}
v_{2 d}(k) \\
v_{2 q}(k)
\end{array}\right]+ \\
+\left[\begin{array}{cc}
0 & \frac{\omega_{s l} L_{M} T}{\sigma L_{2}} \\
\frac{-\omega_{s l} L_{M} T}{\sigma L_{2}} & 0
\end{array}\right]\left[\begin{array}{l}
i_{1 d}(k) \\
i_{1 q}(k)
\end{array}\right]
\end{array}
$$

where: $\omega_{s l}=\omega_{1}-B \omega_{r}$ is the slip frequency and $\sigma=1-L_{M}^{2} /\left(L_{1} L_{2}\right)$.

The rotor voltage which is calculated to guarantee null steady state error by using the deadbeat theory [31] is given by:

$$
\begin{array}{r}
v_{2 d}(k)=\sigma L_{2} \frac{i_{2 d}(k+1)-i_{2 d}(k)}{T}+R_{2} i_{2 d}(k)+ \\
-L_{2} \omega_{s l} i_{2 q}(k)-L_{M} \omega_{s l} i_{1 q}(k)
\end{array}
$$




$$
\begin{array}{r}
v_{2 q}(k)=\sigma L_{2} \frac{i_{2 q}(k+1)-i_{2 q}(k)}{T}+R_{2} i_{2 q}(k)+ \\
+L_{2} \omega_{s l} i_{2 d}(k)+L_{M} \omega_{s l} i_{1 d}(k)
\end{array}
$$

For the active power control, the rotor current reference by using (7) is given by:

$$
i_{2 q}(k+1)=i_{2 q_{r e f}}=-\frac{2 P_{r e f} L_{1}}{3 v_{1} L_{M}}
$$

and for the reactive power control by using (8) is:

$$
i_{2 d}(k+1)=i_{2 d_{r e f}}=-\frac{2 Q_{r e f} L_{1}}{3 v_{1} L_{M}}+\frac{\lambda_{1}}{L_{M}}
$$

where: $P_{r e f}$ is the active power reference and $Q_{r e f}$ is the reactive power reference.

Thus, if the $d$ and $q$ axis voltage components calculated according to equations (10), (11), (12), and (13) are applied to the generator then, the active and reactive power convergence to their respective commanded values will occur in one sampling interval. The desired rotor voltage in the rotor reference frame $\left(\delta_{s}-\delta_{r}\right)$ generates switching signals for the rotor side using either space vector modulation.

Stator currents and voltages, rotor speed and currents are measured to stator flux position $\delta_{s}$ and magnitude $\lambda_{1}$, synchronous frequency $\omega_{1}$ and slip frequency $\omega_{s l}$ estimation.

\subsection{Stator flux estimation}

For a Deadbeat power control, as shown in the equations (10) and (11), it is required to calculate the active and reactive power values, their errors, the stator flux magnitude and position, the slip speed and synchronous frequency.

The stator flux $\vec{\lambda}_{1 \alpha \beta}$ estimation in stationary reference frame is given by:

$$
\vec{\lambda}_{1 \alpha \beta}=\int\left(\vec{v}_{1 \alpha \beta}-R_{1} \vec{i}_{1 \alpha \beta}\right) d t
$$

This expression can be implemented to allow the estimation of the stator flux, even though the induction machine is operating at low speed in a direct torque control system, as shown in [32].

Thus, the stator flux position by using equation (14) is given by: 


$$
\delta_{s}=\arctan \left(\frac{\lambda_{1 \beta}}{\lambda_{1 \alpha}}\right)
$$

The synchronous speed $\omega_{1}$ estimation is:

$$
\omega_{1}=\frac{d \delta_{s}}{d t}=\frac{\left(v_{1 \beta}-R_{1} i_{1 \beta}\right) \lambda_{1 \alpha}-\left(v_{1 \alpha}-R_{1} i_{1 \alpha}\right) \lambda_{1 \beta}}{\left(\lambda_{1 \alpha}\right)^{2}+\left(\lambda_{1 \beta}\right)^{2}}
$$

and the slip speed estimation by using the rotor speed and synchronous speed is:

$$
\omega_{s l}=\omega_{1}-B \omega_{m e c}
$$

The angle between stator and rotor flux is given by:

$$
\delta_{s}-\delta_{r}=\int \omega_{s l} d t
$$

\section{Wireless coding communication}

The proposed wireless control system, shown in Fig. 3, uses LDPC codes [9, 12, 33] to improve system performance and reliability.

The LDPC are $\left(N_{c}, N_{b}\right)$ binary linear block codes that have a sparse parity-check matrix $\mathbf{H}$ that can be described in terms of a Tanner graph [34], where each bit in the codeword corresponds to a variable node and each parity-check equation corresponds to a check node. A check node $j$ is connected to a variable node $k$ whenever the element $h_{j, k}$ in $\mathbf{H}$ is equal to $1[9,34]$.

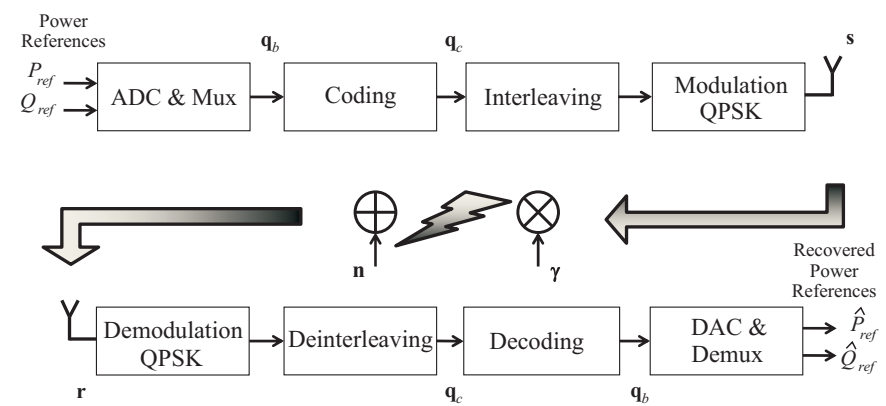

Figure 3. Wireless Coding Communication Diagram. 
Extended Irregular Repeat Accumulate (eIRA) codes [14, 35-38] are a special subclass of LDPC codes that improve the systematic encoding process and generate good irregular LDPC codes for high code rate applications. The eIRA parity-check matrix can be represented by $\mathbf{H}=\left[\begin{array}{ll}\mathbf{H}_{\mathbf{1}} & \mathbf{H}_{\mathbf{2}}\end{array}\right]$, where $\mathbf{H}_{\mathbf{1}}$ is a sparse $\left(N_{m}\right)$ by $\left(N_{c}\right)$ matrix, that can be constructed irregularly by density evolution according to optimal weight distribution [14], and $\mathbf{H}_{\mathbf{2}}$ is the $\left(N_{m}\right)$ by $\left(N_{m}\right)$ dual-diagonal square matrix given by:

$$
\mathbf{H}_{2}=\left[\begin{array}{llllll}
1 & & & & \\
1 & 1 & & & \\
& & \ddots & \ddots & & \\
& & \ddots & 1 & \\
& & & 1 & 1
\end{array}\right]
$$

where: $N_{b}$ is the number of control bits, $N_{c}$ is the number of coded bits and $N_{m}$ is the number of parity bits.

Given the constraint imposed on the $\mathbf{H}$ matrix, the generator matrix can be represented in the systematic form by the $\left(N_{b}\right)$ by $\left(N_{c}\right)$ matrix:

$$
\mathbf{G}=\left[\begin{array}{ll}
\mathbf{I} & \mathbf{\Psi}
\end{array}\right]
$$

where: $\mathbf{I}$ is the identity matrix, $\mathbf{\Psi}=\mathbf{H}_{1}^{\mathrm{T}} \times \mathbf{H}_{2}^{-\mathrm{T}}$ and $\mathbf{H}_{2}^{-\mathrm{T}}$ is the upper triangular matrix given by:

$$
\mathbf{H}_{2}^{-\mathrm{T}}=\left[\begin{array}{rrrrrrr}
1 & 1 & 1 & \cdots & 1 & 1 \\
& 1 & 1 & \cdots & 1 & 1 \\
& & 1 & & 1 & 1 \\
& & & \ddots & \vdots & \vdots \\
& & & & 1 & 1 \\
& & & & 1
\end{array}\right]
$$

The encoding process can be accomplished by first multiplying the control information vector $\mathbf{q}_{b}=\left[q_{b}(1) \cdots q_{b}\left(N_{b}\right)\right]^{T}$ by the sparse matrix $\mathbf{H}_{1}^{\mathrm{T}}$ and then differentially encoding this partial result to obtain the parity bits. The systematic codeword vector $\mathbf{q}_{c}=\left[q_{c}(1) \cdots q_{c}\left(N_{c}\right)\right]^{T}$ can be simply obtained by combining the control information and the parity bits:

$$
\mathbf{q}_{c}=\left[\begin{array}{ll}
\mathbf{q}_{b} & \mathbf{\Psi}
\end{array}\right]
$$


In the transmission process, the codeword vector is then interleaved and Quaternary Phase Shift Keying (QPSK) mapped using Gray code [7], resulting in the symbol vector $\mathbf{s}=\left[s(1) \cdots s\left(N_{s}\right)\right]^{T}$, where $N_{s}$ is the number of transmitted coded control symbols. Afterwards, the coded symbols are filtered, upconverted and transmitted by the wireless fading channel.

Assuming that the channel variations are slow enough that intersymbol interferences (ISI) can be neglect, the fading channel can be modeled as a sequence of zero-mean complex Gaussian random variables with autocorrelation function [7, 39]:

$$
R_{h}(\tau)=J_{0}\left(2 \pi f_{D} T_{S}\right)
$$

where: $J_{0}()$ is the zero-th order Bessel function, $T_{s}$ is the signaling time and $f_{D}$ is the Doppler spread.

Thus, in the receive process, the complex low-pass equivalent discrete-time received signal can be represented by [7]:

$$
\mathbf{r}=\gamma \cdot \mathbf{s}+\mathbf{n}
$$

where: $\mathbf{r}=\left[r(1) \cdots r\left(N_{S}\right)\right]^{T}$ is the received signal vector, $\gamma=\left[\gamma(1) \cdots \gamma\left(N_{S}\right)\right]^{T}$ is the vector of complex coefficients of the channel and $\mathbf{n}=\left[n(1) \cdots n\left(N_{s}\right)\right]^{T}$ is the Additive White Gaussian Noise (AWGN) vector. Note that the above vector multiplication is performed element by element.

Once the transmitted vector $\mathbf{s}$ is estimated, considering perfect channel estimation, the transmitted control bits can be recovered by performing symbol demapping, code deinterleaving and bit decoding.

Decoding can be accomplished by a message passing algorithm [16, 40-42] based on the Maximum A Posteriori (MAP) criterion [9], that exchanges soft-information iteratively between the variable and check nodes. The exchanged messages can be represented by the following Log-Likelihood Ratio (LLR):

$$
L_{c_{k}}=\log \left[\frac{p\left(\mathbf{q}_{c}(k)=0 \mid \mathbf{d}\right)}{p\left(\mathbf{q}_{c}(k)=1 \mid \mathbf{d}\right)}\right]
$$

The LLR message from the $j^{\text {th }}$ check node to the $k^{\text {th }}$ variable node is given by:

$$
L_{r_{j, k}}=2 \operatorname{atanh}\left[\prod_{k^{\prime} \in V_{j \backslash k}} \tanh \left(\frac{L_{q_{k^{\prime}, j}}}{2}\right)\right]
$$


The set $V_{j}$ contains the variable nodes connected to the $j^{\text {th }}$ check node and the set $C_{k}$ contains the check nodes connected to the $k^{t h}$ variable node. $V_{j \backslash k}$ is the set $V_{j}$ without the $k^{\text {th }}$ element, and $C_{k \backslash j}$ is the set $C_{k}$ without the $j^{\text {th }}$ element. The LLR message from the $k^{\text {th }}$ variable node to the $j^{\text {th }}$ check node is obtained by:

$$
L_{q_{k, j}}=L_{\mathcal{c}_{k}}+\sum_{j^{\prime} \in c_{k \backslash j}} L_{r_{j^{\prime}, k}}
$$

and the LLR for the $k^{\text {th }}$ code bit is given by:

$$
L_{Q_{k}}=L_{c_{k}}+\sum_{j \in c_{k}} L_{r_{j, k}}
$$

At the end of each iteration, $L_{Q_{k}}$ provides an updated estimate of the a posteriori LLR of the transmitted coded bit $q_{c}(k)$. If $L_{Q_{k}}>0$, then $q_{c}(k)=1$, else $q_{c}(k)=0$.

\section{Control system performance}

The deadbeat power control strategy, for this analysis, has a sampling time of $0.5 \times 10^{-4}$ s and the DFIG parameters are shown in Appendix. During the period of 1.75-2.0s, the rotor speed was increased from 151 to $226.5 \mathrm{rad} / \mathrm{s}$ to include also the wind variation in the analysis. In the simulations, the active and reactive power references were step changed, respectively, from -100 to $-120 \mathrm{~kW}$ and from 60 to $0 \mathrm{kvar}$ at $1.25 \mathrm{~s}$. At $1.5 \mathrm{~s}$, the references also were step changed from -120 to $-60 \mathrm{~kW}$ and from 0 to -40 kvar. Again, at $1.75 \mathrm{~s}$, the references were step changed from -60 to $-100 \mathrm{~kW}$ and from -40 to $-60 \mathrm{kvar}$. These references are the inputs of the wireless coding power control, shown in Fig. 3, which is analyzed for two different scenarios: an AWGN channel and a more realistic flat fading correlated Rayleigh channel.

The system is evaluated for a frequency flat fading Rayleigh channel with a Doppler spread of $180 \mathrm{~Hz}$. The LDPC coding scheme uses the $(64,800 ; 32,400)$ eIRA code specified in [43], and an ordinary Convolution Coding scheme with a $(171,133)$ generator polynomial with constrain length of 7 is used as reference of performance [9]. Both schemes have code rate of $1 / 2$ and employ a random interleaving of length 64,800 . For simplicity, the number of iterations in the LDPC decoding is limited to 25 . The bit duration is $1.25 \times 10^{-5} \mathrm{~s}$ and each transmitted frame is composed by 32,400 QPSK coded symbols. In Fig. 4 and 5, the final responses of the wireless power control system employing CONV are presented for a typical Noise to Signal Ratio $\left(E_{b} / N_{0}\right)$ of $10 \mathrm{~dB}$. The spikes presented in the responses of the system occur due to the errors in the wireless communication, even with the use of a very efficient error correction scheme. It can be observed that several of these spikes, presented in the reference signals, are followed by the controller and not by others due to the fact that the time response of the controller is not sufficient to follow quick changes caused by destructive effects of the channel in the transmitted signal. 

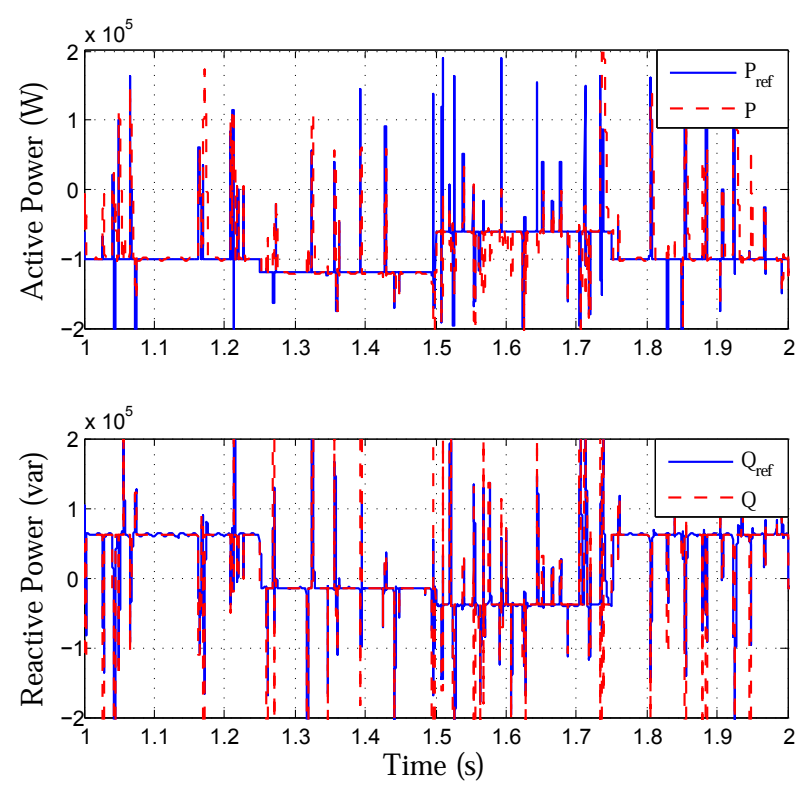

Figure 4. Step Response of Active and Reactive Powers Using CONV Coding in a Flat Fading Channel.
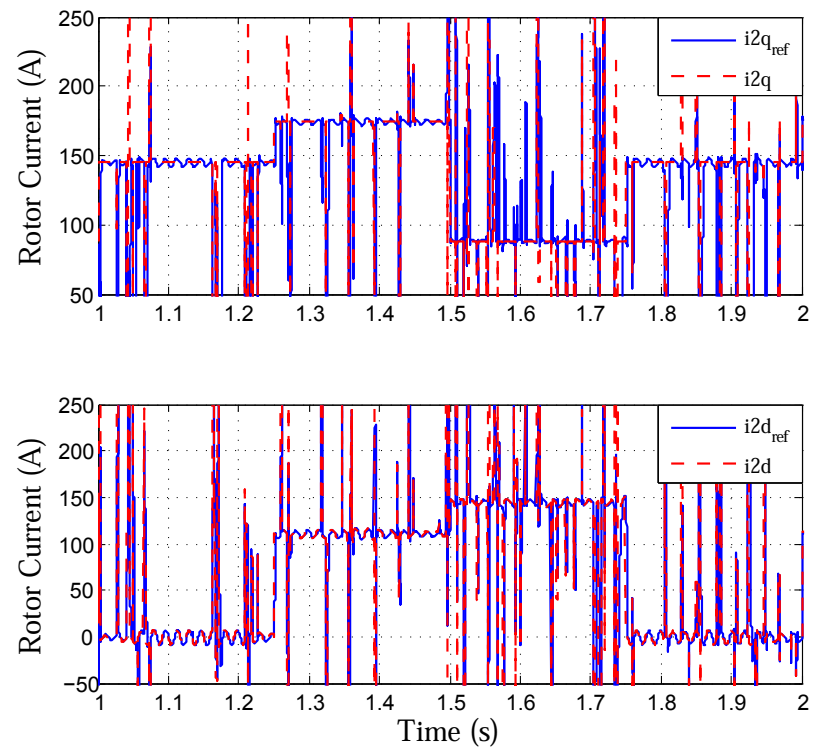

Figure 5. Step Response of Rotor Current $\vec{i}_{2_{d q}}$ Using CONV Coding in a Flat Fading Channel. 
These errors in the control system can permanently damage the aerogenerator, the wind generation system, or even cause a loss of system efficiency, since the machine will not generate its maximum power track at that moment, and additionally, they generate undesirable harmonic components to the power grid. The damage related to wind generation occurs due to the fact that high values of $\frac{d i}{d t}$, as shown in Fig. 6, can completely deteriorate the Insulated Gate Bipolar Transistors (IGBTs) and, consequently, through the power converter, can cause short circuits in rotor and/or stator of the generator.
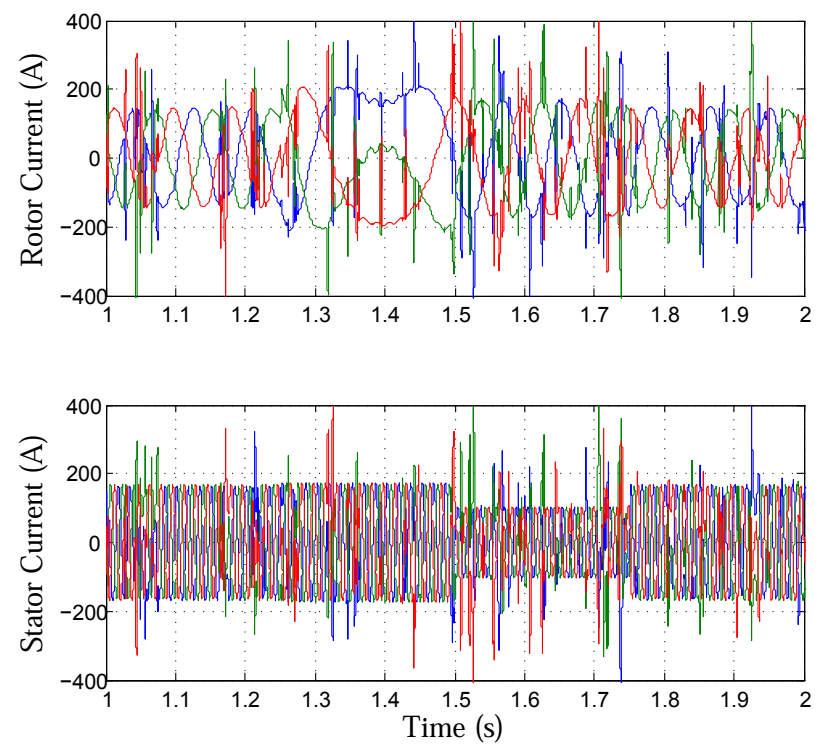

Figure 6. Stator and Rotor Currents Using CONV Coding in a Flat Fading Channel.

Thus, it is necessary to use a wireless control system capable of minimizing the occurrence of these spikes arising from errors caused by the channel distortions. Aiming this, it is highlighted the proposal of using a more robust wireless control system based on LDPC coding. Fig. 7 and 8 show the response of the wireless controller employing the LDPC coding scheme for the same $E_{b} / N_{0}$ of $10 \mathrm{~dB}$ and step reference signals described at the beginning of this section.

The satisfactory performance of the wireless control system can be seen due to the fact the references were perfectly followed by the controller and the inexistence of destructive spikes caused by errors in the wireless transmission system. Additionally, these good functionalities are shown in Fig. 9, where the stator currents present expected waveforms for an good operational functionality. 

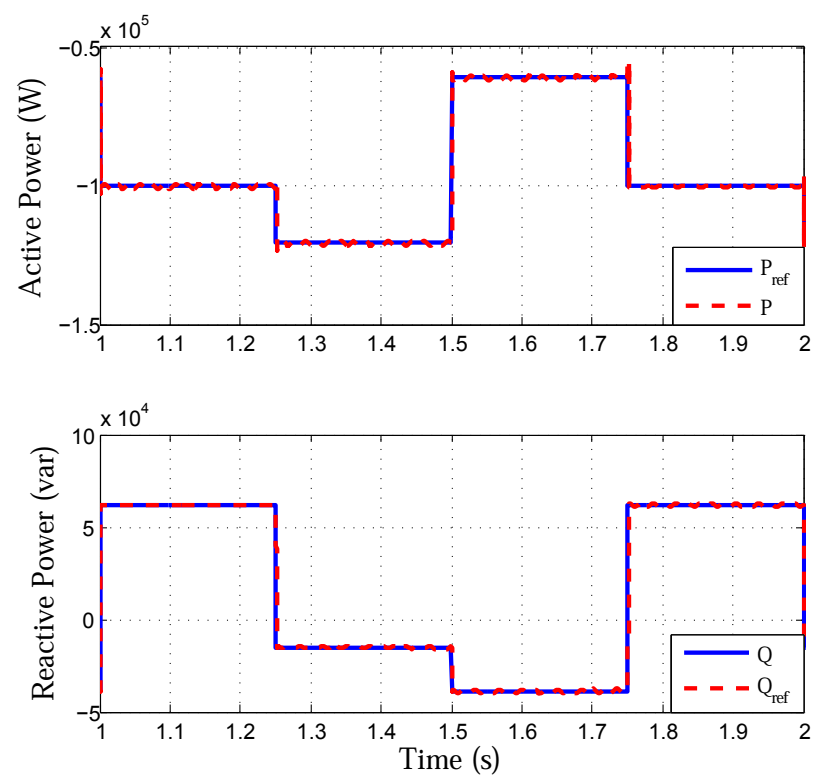

Figure 7. Step Response of Active and Reactive Powers Using LDPC Coding in a Flat Fading Channel.
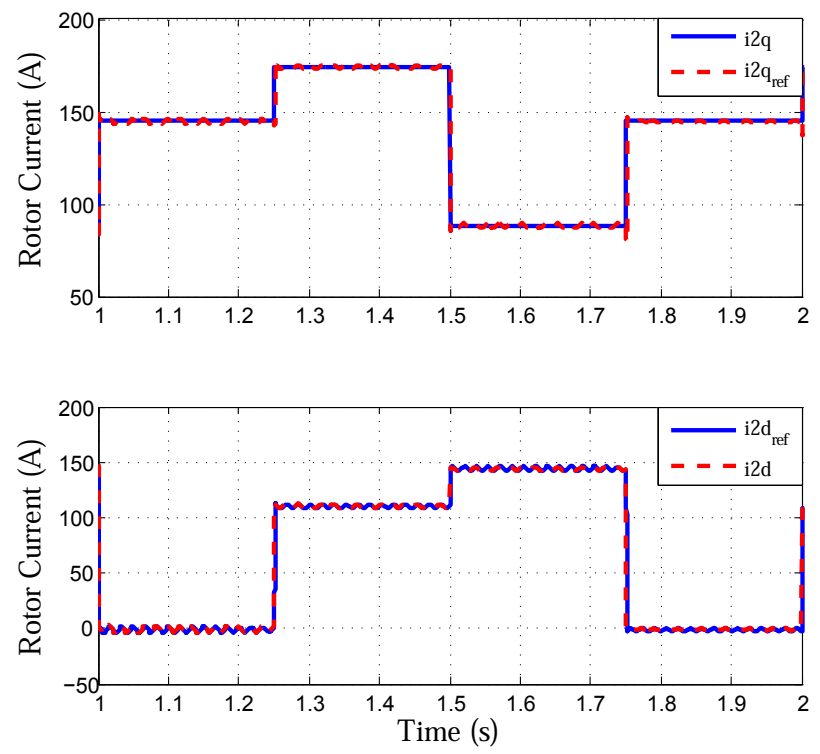

Figure 8. Step Response of Rotor Current $\vec{i}_{2_{d q}}$ Using LDPC Coding in a Flat Fading Channel. 

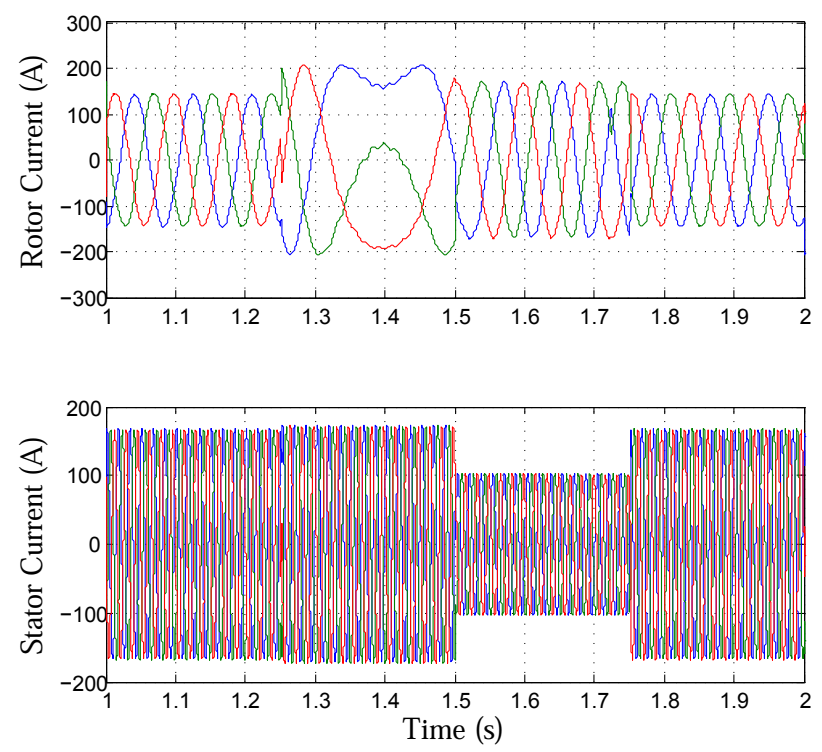

Figure 9. Stator and Rotor Currents Using LDPC Coding in a Flat Fading Channel.

To complete the analysis, it is evaluated the performance of the proposed wireless coded deadbeat power control system for many values of $E_{b} / N_{0}$ in a flat fading channel. In Fig. 10, a comparison of performance for No Coding, CONV, and LDPC schemes is presented. As expected, the performance of LDPC is significantly superior than CONV. As pointed out, the performance improvement of LDPC over CONV for a BER of $10^{-5}$ is approximately $26.8 \mathrm{~dB}$, demonstrating the good performance of LDPC in this channel condition.

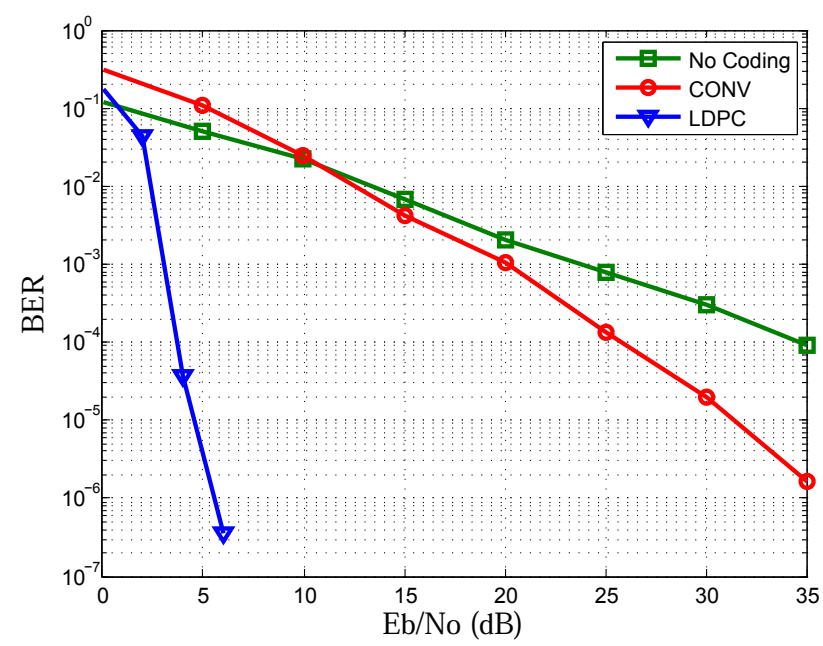

Figure 10. Performance Comparison for Different Coding Schemes in Flat Fading Channel (180 Hz - QPSK). 
Table 1 shows the number and percentage of phase inversions, corresponding to $P_{\text {ref }}$ and $Q_{r e f}$ references, presented in the recovered control signal for both scenarios. It can be seen that LDPC coding requires a significant lower $E_{b} / N_{0}$ to present the same order of phase inversions as CONV.

For a low BER as $10^{-5}$, there are some changes in the active and reactive power references that can cause serious problems in the generator, and consequentially, in the energy plant. However, the use of LDPC coding can reduce notably this number for a typical $E_{b} / N_{0}$ in real systems and can improve considerably system robustness to the channel impairments. For instance, a system operating with an $E_{b} / N_{0}$ of $10 \mathrm{~dB}$ employing CONV will fail dramatically, while a system using LDPC coding will be free of communication errors, showing the real operational viability of the proposed control technique in wind power plants.

\begin{tabular}{cccc}
\hline $\begin{array}{c}\text { Coding } \\
\text { Scheme }\end{array}$ & $\begin{array}{c}\text { Bit Error } \\
\text { Rate }\end{array}$ & $\begin{array}{c}\text { Eb/No } \\
(\mathbf{d B})\end{array}$ & $\begin{array}{c}\text { Number of } \\
\text { Inversions }\end{array}$ \\
\hline CONV & $10^{-3}$ & 20.0 & $167(0.087 \%)$ \\
CONV & $10^{-4}$ & 25.5 & $21(0.01 \%)$ \\
CONV & $10^{-5}$ & 31.5 & $4(0.002 \%)$ \\
LDPC & $10^{-3}$ & 3.20 & $188(0.098 \%)$ \\
LDPC & $10^{-4}$ & 3.80 & $26(0.013 \%)$ \\
LDPC & $10^{-5}$ & 4.70 & $1(0.0005 \%)$ \\
\hline
\end{tabular}

Table 1. Control Inversion in a Flat Fading Channel.

\section{Conclusion}

This work proposes a wireless coding control system using a deadbeat controller applied to a doubly-fed induction aerogenerator for smart grid applications. An analysis for different coding schemes shows that, even for a relatively low BER, the power reference changes can occur and it can be very dangerous for the generator and the energy plant. However, the use of LDPC coding improves significantly the robustness of the system in severe noise and fading channel situations, eliminating the occurrence of errors in the active and reactive power references for operational conditions.

In addition, it is noteworthy that the errors generated in the wireless transmission cannot be easily removed without using advanced FEC coding techniques similar to the one proposed in this work without increasing significantly the quantity of retransmissions, that should be controlled, for instance, by an Automatic Repeat Request (ARQ) scheme implemented in an upper communication layer. On the other hand, depending on channel conditions, even employing an efficient ARQ scheme, the latency of the system can increase dramatically without a similar technique as the proposed in this paper. Alternatively, as a future work, other types of codification and modulation can be explored. 


\section{Appendix}

Doubly-fed induction generator parameters are: [44]:

$R_{1}=24.75 \mathrm{~m} \Omega ; R_{2}=13.3 \mathrm{~m} \Omega ; L_{M}=14.25 \mathrm{mH} ; L_{l 1}=284 \mu \mathrm{H} ; L_{l 2}=284 \mu \mathrm{H} ; J=2.6 \mathrm{Kg} \cdot \mathrm{m}^{2}$; $B=2 ; P_{N}=149.2 \mathrm{kVA} ; V_{N}=575 \mathrm{~V}$.

where: $R$ - Winding resistance, $L_{M}$ - Mutual inductance, $L_{l}$ - Dispersion inductance, $J$ Moment of inertia, $B$ - Number of pair poles, $P_{N}$ - Nominal Power, $V_{N}$ - Nominal Voltage. The subscripts 1 and 2 represent the stator and rotor parameters, respectively.

\section{Author details}

C. E. Capovilla ${ }^{1}$, A. J. Sguarezi Filho ${ }^{1}$,

I. R. S. Casella ${ }^{1}$ and E. Ruppert ${ }^{2}$

1 Universidade Federal do ABC - UFABC, Brazil

2 Universidade Estadual de Campinas - Unicamp, Brazil

\section{References}

[1] M. Godoy SimÃţes and Felix A. Farret. Renewable Energy Systems with Induction Generators. CRC Press, 2004.

[2] J. Blau. Europe plans a north sea grid. IEEE Spectrum, pages 08-09, March 2010.

[3] M. Glinkowski, J. Hou, and G. Rackliffe. Advances in wind energy technologies in the context of smart grid. Proceedings of the IEEE, 99(6):1083-1097, June 2011.

[4] J. Wang, X. Du, and X. Zhang. Comparison of wind power generation interconnection technology standards. Asia-Pacific Power and Energy Engineering Conference, March 2011.

[5] R. Strzelecki and G. Benysek. Power Electronics in Smart Electrical Energy Networks. Springer-Verlag, 2008.

[6] T. J. Li. Low complexity capacity approaching schemes: Design, analysis, and applications. Ph.D. dissertation, Texas AM Univ., 2002.

[7] J. G. Proakis. Digital Communications. MCGraw-Hill, 2001.

[8] J. Jiang and K. R. Narayanan. Iterative soft decision decoding of Reed Solomon. IEEE Communications Letters, 8:244-246, 2004.

[9] S. Lin and D. J. Costello. Error control coding. Prentice Hall, 2004.

[10] C. Berrou, A. Glavieux, and P. Thitimajshima. Near Shannon limit error-correcting coding and decoding: Turbo-codes. IEEE International Communications Conference, pages 1064-1070, 1993.

[11] J. Chen and A. Abedi. Distributed turbo coding and decoding for wireless sensor networks. IEEE Communications Letters, 15:166-168, 2011. 
[12] R. G. Gallager. Low-Density Parity-Check Codes. Cambridge, 1963.

[13] D. J. C. MacKay and R. M. Neal. Near Shannon limit performance of low-density parity-check codes. IET Electronics Letters, 32:1645-1646, 1996.

[14] Y. Zhang, W. E. Ryan, and Y. Li. Structured eIRA codes with low floors. Proceedings of the International Symposium on Information Theory, pages 174-178, September 2005.

[15] E. C. Shannon. A mathematical theory of communication. Bell System Technical Journal, 27:379-423, 1948.

[16] T. Richardson, A. Shokrollahi, and R. Urbanke. Design of capacity-approaching low-density parity-check codes. IEEE Transactions on Information Theory, 47:619-637, February 2001.

[17] IEEE. Standard for local and metropolitan area networks, part 16: Air interface for fixed and mobile wireless access systems. IEEE Std. 802.16-2004, 2004.

[18] M. Adamowicz, R. Strzelecki, Z. Krzeminski, J. Szewczyk, and L. Lademan. Application of wireless communication to small WECS with induction generator. IEEE Mediterranean Electrotechnical Conference, pages 944-948, June 2010.

[19] M. Adamowicz, R. Strzelecki, Z. Krzeminski, J. Szewczyk, and L. Lademan. Wireless short-range device for wind generators. 12th Biennial Baltic Electronics Conference, pages 1736-3705, November 2010.

[20] J. F. Manwell, J. G. McGowan, and A. L. Rogers. Wind Energy Explained: Theory, Design and Application. 2 edition, 2010.

[21] R. Datta and V. T. Rangathan. Variable-speed wind power generation using doubly fed wound rotor induction machine - A comparison with alternative schemes. IEEE Trans. on Energy Conversion, 17(3):414-421, September 2002.

[22] Arantxa Tapia, Gerardo Tapia, J. Xabier Ostolaza, and JosÃ̃l' RamÃşn SÃąenz. Modeling and control of a wind turbine driven doubly fed induction generator. IEEE Trans. on Energy Conversion, (194-204), June 2003.

[23] Zhang Xin-fang, XU Da-ping, and LIU Yi-bing. Predictive functional control of a doubly fed induction generator for variable speed wind turbines. IEEE World Congress on Intelligent Control and Automation, June 2004.

[24] J. Morren and S. W. H. Haan. Ridethrough of wind turbines with doubly-fed induction generator during a voltage dip. IEEE Transactions on Energy Conversion, 20(2):435-441, June 2005.

[25] Ji. Guo, X. Cai, and Y. Gong. Decoupled control of active and reactive power for a grid-connected doubly-fed induction generator. Third International Conference on Electric Utility Deregulation and Restructuring and Power Technologies, pages 2620 - 2625, April 2008 . 
[26] Z. Li, F. Zheng, Y. Wu, and H. Gao. Offshore wind farm construction platform jack up control system. World Non-Grid-Connected Wind Power and Energy Conference, pages 24-26, September 2009.

[27] O. Anaya-Lara, N. Jenkins, and J. R. McDonald. Communications requirements and technology for wind farm operation and maintenance. IEEE International Conference on Industrial and Information Systems, pages 173-178, August 2006.

[28] C. Wanzhi, T. Zhiyong, Z. Quangui, and C. Liang. Research of wireless communication based on lonworks for wind turbine control system. IEEE International Conference on Energy and Environment Technology, pages 787-789, October 2009.

[29] D. W. Novotny and T. A. Lipo. Vector Control and Dynamics of AC Drives. Clarendon Press Oxford, 1996.

[30] A. J. Sguarezi Filho and E. Ruppert. A deadbeat active and reactive power control for doubly-fed induction generators. Electric Power Components and Systems, 38(5):592-602, 2010.

[31] G. F. Franklin, J. D. Powel, and M. Workman. Digital Control of Dynamic Systems. Addison-Wesley Publishing Company, 1994.

[32] A. J. Sguarezi Filho and E. Ruppert. The complex controller for three-phase induction motor direct torque control. SBA - Controle e automa $\tilde{A} \breve{g} \tilde{A} \check{c} o$, 20(2):256-262, 2009.

[33] Y. Zhang and W. E. Ryan. Toward low LDPC-code floors: a case stud. IEEE Transactions on Communications, 57(6):1566-1573, June 2009.

[34] R. M. Tanner. A recursive approach to low complexity codes. IEEE Transactions on Information Theory, 27(5):533-547, September 1981.

[35] H. Jin, A. Khandekar, and R. J. McEliece. Irregular repeat-accumulate codes. Proc. Int. Symp. Turbo Codes and Related Topics, pages 1-5, September 2000.

[36] M. Yang and W. E. Ryan. Lowering the error-rate floors of moderate length high-rate irregular LDPC codes. Int. Symp. Information Theory, 2:237, July 2003.

[37] M. Yang, W. E. Ryan, and Y. Li. Design of efficiently encodable moderate-length high-rate irregular LDPC codes. IEEE Transactions on Communications, 52(4):564-571, April 2004.

[38] J. Kim, A. Ramamoorthy, and S. Mclaughlin. The design of efficiently-encodable rate-compatible LDPC codes. IEEE Transactions on Communications, 57:365-375, 2009.

[39] A. Barbieri, A. Piemontese, and G. Colavolpe. On the ARMA approximation for frequency-flat rayleigh fading channels. IEEE International Symposium on Information Theory, pages 1211-1215, June 2007. 
[40] T. Richardson and R. Urbanke. The capacity of low-density parity check codes under message-passing decoding. IEEE Transactions on Information Theory, 47:599-618, February 2001.

[41] L. Dinoi, F. Sottile, and S. Benedetto. Design of versatile eIRA codes for parallel decoders. IEEE Transactions on Communications, 56(12):2060-2070, 2008.

[42] B. Shuval and I. Sason. On the universality of LDPC code ensembles under belief propagation and $\mathrm{ml}$ decoding. IEEE 26th Convention of Electrical and Electronics Engineers, pages 355-359, 2010.

[43] ETSI. DVB-S.2. Standard Specification, pages 302-307, March 2005.

[44] A. J. Sguarezi Filho, M. E. de Oliveira Filho, and E. Ruppert. A predictive power control for wind energy. IEEE Transactions on Sustainable Energy, 2(1):97-105, January 2011. 
